\title{
EVOLUCIÓN DE LA INGENIERÍA CIVIL EN LA CIUDAD DE GUAYAQUIL DURANTE EL SIGLO XX
}

\author{
EVOLUTION OF CIVIL ENGINEERING IN THE CITY OF GUAYAQUIL IN THE \\ TWENTIETH CENTURY
}

\section{NANCY VARELA TERREROS ${ }^{1}$}

Instituto de Investigación de la Facultad de Ingeniería de la Universidad Católica de Santiago de Guayaquil (IIFIUC). nancy.varela@cu.ucsg.edu.ec

RESUMEN

El objetivo principal de esta investigación ha sido el de caracterizar los hitos esenciales en el crecimiento de la ingeniería civil, en todas sus ramas, que se produjeron durante el Siglo XX, en la ciudad de Guayaquil y su vinculación con el desarrollo actual de la ingeniería civil en la ciudad. Para ello se visitaron lugares estratégicos de la ciudad, que marcaron esta evolución o crecimiento. Se recopiló información bibliográfica de interés y se realizaron entrevistas a fondo con personas vinculadas con la carrera. Los principales resultados obtenidos evidenciaron que: junto con el crecimiento poblacional, la ciudad pasó de tener viviendas de madera y mixtas a viviendas de hormigón, con edificios de hasta 30 pisos; las calles empedradas han sido sustituidas por calles pavimentadas. En cuanto al servicio de agua, la ciudad evolucionó y se expresa que: el agua potable se provee por tubería al interior de las casas en casi la totalidad de la ciudad; el agua servida es recogida por canalización, y en algunos sitios es tratada antes de su descarga al cuerpo receptor; sin embargo, persisten letrinas y pozos ciegos. El agua lluvia es enviada por ductos cajones y tuberías a sus cuerpos receptores. La investigación permitió estimar que el crecimiento de la ciudad, posiblemente, seguirá presentándose en los mismos ámbitos en que lo ha hecho hasta ahora, puesto que de momento dichos ámbitos siguen siendo de interés para la sociedad. La evolución de la ingeniería civil es producto de las necesidades de la población, por lo que nuevas necesidades implicarán nuevos ámbitos de desarrollo. En la actualidad se cuenta con enseñanza de Ingeniería Civil en cinco universidades en la ciudad, lo que hace que sean profesionales propios los que intervengan en el desarrollo de Guayaquil.

PALABRAS CLAVE: ingeniería civil, vivienda, desarrollo, universidad, agua, servicios.

\section{ABSTRACT}

The main objective of this research was to characterize the essential milestones in the growth of all the branches of the civil engineering field, which took place in the twentieth century, in the city of Guayaquil and how this relates with the current development of civil engineering in the city. With this purpose, strategic places of the city that marked this evolution or growth were visited. Bibliographic information of interest was collected and in-depth interviews were made to people associated with the field. The main results showed that: along with population growth, the city went from having wooden and mixed housing to concrete housing, with buildings up to 30 floors; the cobbled streets have been replaced by paved streets. As for the water service, the city evolved and it was stated that: drinking water is supplied by pipes inside the houses in almost all of the city; the wastewater is collected through pipelines, and in some places it is treated before discharging it to the receptor; however, latrines and septic tanks still exist. Rain water is sent through crate pipelines and pipelines to receptors. The research allowed to estimate that the growth of the city will possibly continue in the same areas in which it has evolved so far because these areas are still of interest to society. The evolution of civil engineering is the product of population needs, thus, as new requirements emerge, new areas of development will materialize. Currently, civil engineering is thought in five universities in the city, which implies that these professionals will be the ones involved in the development of Guayaquil.

KEYWORDS: civil engineering, housing, development, university, water, services. 


\section{INTRODUCCIÓN \\ IDENTIFICACIÓN DEL PROBLEMA Y JUSTIFICACIÓN}

No se ha realizado hasta nuestros días un estudio sobre la historia de la Ingeniería Civil en nuestra ciudad que señale qué se ha hecho desde el inicio de la profesión, cuáles han sido sus principales hitos, cuál ha sido la principal tendencia de crecimiento, los factores que han incidido en estos cambios o han facilitado la consecución de hitos, ni quienes han sido los protagonistas o desarrolladores de estos hitos. El conocimiento de esta historia, dentro de un estudio sobre la Evolución de la Ingeniería Civil en la ciudad de Guayaquil durante el Siglo XXI, facilitará el desarrollo y planificación del crecimiento de Guayaquil en el corto y mediano plazo.

Este estudio puede ser un aporte a las futuras generaciones de ingenieros civiles, para que se generen nuevas soluciones basadas en aquellas soluciones que ya han sido implementadas y funcionan con éxito hasta nuestros días, así como para resolver problemas nuevos, tomando en cuenta las ideas acertadas y los errores del pasado. Además, este estudio ofrecería a los estudiantes y profesores de la carrera, referentes esenciales para su desarrollo futuro y un aporte al fortalecimiento del conocimiento en general sobre esta parte de la historia y los valores culturales que suponen.

MATERIALES Y MÉTODOS

El presente estudio se sustentó en las siguientes preguntas de investigación: ¿Cuál era el estado de la ingeniería civil guayaquileña en sus diversas ramas a inicios del siglo XX? ¿Cómo evolucionó la Carrera de Ingeniería Civil durante el siglo XX? En función de lo anterior, los factores sociales, económicos y políticos actuales ien qué dirección podrían generar un crecimiento de la ingeniería civil?

OBJETIVO GENERAL

Caracterizar los hitos esenciales en el crecimiento de la ingeniería civil, en todas sus ramas, que se produjeron durante el Siglo XX, en la ciudad de Guayaquil y su vinculación con el desarrollo actual de la ingeniería civil en la ciudad.

\section{OBJETIVOS ESPECÍFICOS}

- Caracterizar las obras más importantes de la ingeniería civil guayaquileña en todas sus ramas, destacando sus rasgos más relevantes y novedosos para su época.
- Valorar la importancia histórica y cultural de las obras de ingeniería civil en la etapa seleccionada (Siglo XX)

- Identificar la influencia de culturas occidentales, orientales y propias presentes en la conceptualización, diseño y construcción de las obras

- Destacar los aportes de las más importantes figuras en la ingeniería civil en el pasado siglo

ENFOQUE METODOLÓGICO

Se realizó un estudio histórico con enfoque cualitativo. Debido a la amplitud del campo de acción en el que se podía estudiar el tema, se sistematizó la información en diversas fuentes, con esa finalidad se diseñaron las siguientes etapas:

- Recopilación bibliográfica sobre la historia de la ciudad, a nivel de vivienda, servicios básicos e infraestructura urbana. Esta recopilación incluyó visitas a sitios estratégicos en que se constataba el desarrollo de los temas estudiados: vivienda, servicio de agua, urbanismo, enseñanza de la carrera.

- En función de la información recopilada, se contactó dentro de los profesionales nacionales y extranjeros radicados en el Ecuador, a aquellos que pudieron ofrecer información de interés para el presente proyecto. Estos profesionales fueron principalmente profesionales vinculados con la enseñanza de ingeniería civil en nuestra ciudad, y profesionales responsables por la provisión de los servicios básicos. A ellos se les realizó una entrevista a profundidad, con la que se pudo obtener información que luego tuvo soporte con visitas en sitio.

\section{RESULTADOS}

El resultado de la presente investigación se puede dividir en diferentes áreas:

Desarrollo urbano y vivienda. La ciudad evolucionó durante toda su historia, a partir de su fundación en el S XVI, de una vivienda autóctona de los aborígenes del Guayas, hasta aquella que copiaba directamente las formas de construcción española. El siglo XX arrancó con viviendas de madera y mixtas, producto de la mezcla cultural - en la que prevaleció la cultura española - y los materiales que podían conseguirse en el sitio, que estaban asentadas en unas pocas calles alrededor de los cerros Santa Ana y El Carmen, y a la orilla del río Guayas. Algunas 
de ellas estaban pavimentadas, y la mayor parte de la construcción era de viviendas de madera de una y dos plantas. Desde ahí, la ciudad evolucionó hasta fines del siglo XX a viviendas únicamente de estructura de hormigón y estructura metálica. Las únicas viviendas de construcción mixta o de madera son las construcciones antiguas. También sobreviven viviendas marginales que poco a poco van desapareciendo con la actuación de las autoridades correspondientes. En vialidad, casi toda la ciudad se encuentra pavimentada.

- Servicio de agua. La ciudad evolucionó del aprovisionamiento de agua mediante un servicio fluvial sin tratamiento, que venía de fuera de la ciudad, hasta la provisión de agua tratada y por tubería, hasta el interior de las viviendas. En cuanto al alcantarillado sanitario y pluvial, el cambio se produjo desde la recolección de desperdicios humanos a domicilio por personas que realizaban este trabajo como medio de sustento, hasta la canalización en la mayor parte de la ciudad. Las zonas no conectadas al final del siglo XX tenían letrinas o pozos ciegos. El agua lluvia pasó de ser enviada de modo natural a sus cuerpos receptores, a ser canalizada por medio de ductos y tuberías instalados a todo lo largo de la ciudad.

- Enseñanza de Ingeniería Civil. Hasta inicios del siglo anterior, en la ciudad de Guayaquil, únicamente habían trabajado en el desarrollo de nuestra ciudad, desde el campo de la Ingeniería Civil, profesionales extranjeros, principalmente europeos y estadounidenses. Esto, debido al hecho de que en Guayaquil no existían centros de educación superior por aquellos días.

- La primera universidad de la ciudad fue la Universidad de Guayaquil, fundada durante el Siglo XIX. Esta institución no contaba en sus inicios con la carrera de Ingeniería Civil, que abrió sus puertas en 1933. Luego de ello, en la medida en que fueron creándose centros de educación superior, aparecieron otros centros de enseñanza de la carrera, en el siguiente orden cronológico: Universidad Católica de Santiago de Guayaquil, Universidad Laica Vicente Rocafuerte, Escuela Superior Politécnica del Litoral, y en el siglo XXI, Universidad de Especialidades Espíritu Santo.

DISCUSIÓN

Al inicio del siglo anterior la ingeniería civil guayaquileña dependía exclusivamente del aporte de profesionales extranjeros, y su crecimiento se daba principalmente en las áreas de vivienda y urbanismo, si se considera que no existían en ese momento ningún tipo de obra de infraestructura urbana ni de ninguna otra naturaleza, en construcción (INEC, 1996). Los pocos puentes existentes eran de madera y servían para cruzar esteros al interior de la ciudad (Terreros, 1988). No había obras de ingeniería hidráulica, carreteras, calles pavimentadas, no se realizaba tratamiento de potabilización de agua, ni de desinfección de aguas servidas. Las viviendas eran de madera, y se había tenido que reconstruir la ciudad varias veces, porque al ser construidas en madera debían ser reconstruidas luego de los grandes incendios que azotaron Guayaquil (Estrada Julio, 2005).

En este contexto, el presente trabajo presenta como hitos de la ingeniería, que marcaron el desarrollo de la ciudad, el cambio de la vivienda de madera - que es un material inflamable y que hasta nuestros días no ha sido utilizado como material para construcción en edificios altos - hasta viviendas con estructura metálica y de hormigón, con mampostería de bloques de diferentes tipos (Álava, J.; Baquerizo, H.; Hagó, F., 1976). Este cambio permite dos grandes ventajas: la resistencia al fuego y el crecimiento vertical.

Otro hito importante ha sido el cambio en el abastecimiento de agua, que el día de hoy es provista en las casas de los pobladores de la ciudad, luego de un tratamiento que cumple con normas internacionales, mientras que al inicio del siglo XX el agua debía ser conseguida en barriles o baldes. Se ha cambiado asimismo la captación del agua cruda. En la actualidad, se obtiene el agua del río Daule, afluente del Guayas, que tiene capacidad para abastecer a toda la ciudad (Envirosoft, 2005).

En vialidad y desarrollo urbano también se ha conseguido hitos de crecimiento, no sólo por las grandes autopistas existentes sino porque poco a poco toda la ciudad está alcanzando un nivel total de pavimentación.

Un eje transversal a este progreso de la ciudad ha sido la enseñanza de la Ingeniería Civil, presente en cinco instituciones diferentes. Cada una de estas instituciones tiene un diferente enfoque aun cuando persiguen un mismo fin que es la producción de Ingenieros Civiles que puedan generar mayor progreso según lo requiera la evolución de la sociedad. Con los nuevos profesionales que realizaron sus estudios de tercer nivel en nuestra ciudad, se 
empezaron a sentar las bases del crecimiento de Guayaquil, en términos de desarrollo de proyectos de infraestructura, vivienda, y provisión de servicios que se brindan hasta hoy. De hecho, actualmente son profesionales guayaquileños, con títulos obtenidos en Guayaquil, los que dirigen y protagonizan este crecimiento.

Es de recalcar que el crecimiento de la ciudad, producido durante el siglo anterior, ha sido producto de las necesidades presentes en ese momento. Es criterio de la autora que el crecimiento actual de la ingeniería civil será dirigido hacia la solución de problemas de escasez de vivienda, generación de carreteras, explotación de recursos hídricos y cuidado en la sostenibilidad del medio ambiente. Estos hilos conductores podrían generar mayor asimismo otro eje transversal, que sería la enseñanza de la ingeniería civil con enfoque en estos temas.

CONCLUSIONES

Con la evolución de Guayaquil durante el siglo $\mathrm{XX}$, que inició como una pequeña ciudad de $\mathrm{po}^{-}$ cas cuadras junto al río Guayas hasta la ciudad de cuatro millones de habitantes de nuestros días, evolucionó simultáneamente la Ingeniería Civil. Las necesidades del crecimiento poblacional, se cristalizaban en ámbitos como la construcción, abastecimiento de agua potable, recolección de aguas servidas y aguas lluvias, pavimentación, vivienda, y una larga lista de requerimientos, para los cuales hacía falta ingenieros civiles.

Estos requerimientos se tradujeron, entre otras cosas, en la creación de la carrera de Ingeniería Civil, en cinco universidades de la ciudad: Universidad de Guayaquil (1933), Universidad Católica de Santiago de Guayaquil (1962), Universidad Laica Vicente Rocafuerte (1966) y Escuela Superior Politécnica del Litoral (1989) y Universidad de Especialidades Espíritu Santo, en Samborondón (2008).

Estas cinco universidades, han producido profesionales que desde hace varias décadas lideran el crecimiento de la Ingeniería Civil en Guayaquil, desde el ámbito de la empresa pública y privada, en todos los sectores mencionados. Gracias a estas universidades, y luego de que su historia la hiciera depender de profesionales extranjeros, la ciudad empezó a tener técnicos que generaran sus propias ideas para definir su crecimiento.

Uno de los campos en que se ha verificado este crecimiento es en el abastecimiento de agua potable y recolección de aguas servidas y aguas lluvias. En cien años, la ciudad pasó de recibir agua no tratada traída en balsa desde fuera de la ciudad (Pérez R., 2002), hasta tener agua tratada, con niveles de exigencia internacionales, por tubería en el 61.6 por ciento de domicilios (Envirosoft, 2005), con una meta inmediata para el presente siglo de agua por tubería en el 100\% de la ciudad. En cuanto a alcantarillado sanitario, la ciudad pasó de tener un sistema de recolección casi artesanal, representado por el abromiquero que recogía desechos humanos en las viviendas (Estrada Jenny, 2010) hasta tener un sistema de recolección en la mayor parte de la ciudad, por tubería. Están desapareciendo poco a poco las letrinas y pozos ciegos (INEC, 2006), y es meta al corto plazo también la cobertura del $100 \%$. Hay, a la presente fecha, un estudio de pre factibilidad para un nuevo sistema de tratamiento de aguas servidas que cubrirá a toda la ciudad. Los estudios correspondientes han sido realizados por profesionales nacionales.

En la actualidad, los servicios de agua potable, alcantarillado sanitario y alcantarillado pluvial son manejados casi en su totalidad por profesionales locales.

En cuanto a tratamiento de agua potable, el Complejo La Toma, cuyas bases conceptuales fueron sentadas por la compañía extranjera J.G. White Ltd. (Estrada Julio, 1995) y que fue inaugurado en el año 1950, funcionó conjuntamente con la planta La Lolita hasta 1975 (Estrada Julio, 1995). Desde entonces, hasta nuestros días, es la única planta de tratamiento de agua potable para nuestra ciudad.

El alcantarillado sanitario evolucionó desde sistemas de recolección de excretas en barriles que se vaciaban en el río (Álava, Baquerizo y Hago, 1976) que funcionaron desde el siglo XVII y con los que la ciudad vio nacer el siglo XX (PP El Verdadero, 2011), hasta sistemas de recolección y tratamiento de aguas servidas que abastecen a casi toda la ciudad.

Esta participación del profesional local también es palpable en cuanto a desarrollo de vivienda, puesto que las urbanizaciones y construcciones actuales, son en su mayor parte realizadas por empresas y profesionales independientes, provenientes de nuestra ciudad, y en algunos casos, de otras zonas de nuestro país. Lo mismo puede decirse en cuanto a pavimentación.

De lo antedicho se desprende:

- La evolución de la ingeniería civil se ha dado como resultado del crecimiento poblacional de la ciudad y de sus cambiantes necesidades a lo largo del tiempo. 
- Los principales campos en que este desarrollo ha sido palpable son: vivienda, servicios de agua, pavimentación e infraestructura urbana.

- Esta evolución de la ingeniería civil hizo necesaria la creación de la carrera de Ingeniería Civil en diversas universidades de la ciudad. La enseñanza dentro de dichas universidades, debió acomodarse también a las necesidades de la ciudad y a los cambios tecnológicos globales.

- En vista del hecho de que la población va a seguir creciendo, y con ella, aumentarán las necesidades de la ciudad, se prevé que la Ingeniería Civil también evolucionará a la par de dichas necesidades, y de los avances tecnológicos que se presenten en el futuro.

\section{REFERENCIAS BIBLIOGRÁFICAS}

Âlava, J.; Baquerizo, H.; Hagó, F. (1976): Evolución Histórica de la Vivienda Dentro del Marco del Desarrollo Urbano en la Región de Guayaquil. Guayaquil: UCSG.

Argudo, J. (2011): Proyecto Radius - Herramientas de Evaluación de Riesgo para el Diagnóstico de Zonas Urbanas contra Desastres Sísmicos - Resumen Ejecutivo. Guayaquil: Universidad Católica de Santiago de Guayaquil.

Cualla López, Ricardo (2008): Elementos de Diseño para Acueductos y Alcantarillados (Segunda Edición) Bogotá: Editorial Escuela Colombiana de Ingeniería.

Donoso Pareja, Miguel (1982): Nunca Más El Mar (Primera Edición). Quito: Editorial El Conejo.

Embajada de Italia en Ecuador (2008): Los Italianos en la Mitad del Mundo (Primera Edición). Quito: Ediecuatorial.

Envirosoft Cia. Ltda. (2005): Plan Maestro de Agua Potable para la Ciudad de Guayaquil. Guayaquil: Interagua.

Envirosoft Cia. Ltda. (2005): Plan Maestro de Alcantarillado Sanitario para la Ciudad de Guayaquil. Guayaquil: Interagua.

Envirosoft Cia. Ltda. (2005): Plan Maestro de Drenaje Pluvial para la Ciudad de Guayaquil. Guayaquil: Interagua.

Envirosoft Cia. Ltda. (2005): Estudio de Impacto Ambiental para el Proyecto de Expansión del Sistema de Alcantarillado Sanitario Guasmo Sur. Guayaquil: Interagua.

Escalante, G., Solano De La Sala, M. (2003): Bucay, Un Recurso Turístico Para Turismo De Eco-Aventura. Guayaquil: ESPOL.

Estrada, J. (2010): Del Tiempo de la Yapa (Quinta edición). Guayaquil: Diario El Universo.
Gómez, J. (2005): Historia del Malecón de Guayaquil (Primera Edición). Guayaquil: Archivo Histórico del Guayas del Banco Central del Ecuador.

Hoyos, M.; Avilés, E. (2006): Guayaquil Memorias Urbanas (Primera Edición). Guayaquil: M.I. Municipalidad de Guayaquil.

Hoyos, M.; Avilés, E. (2006): Historia de Guayaquil (Primera Edición). Guayaquil: M.I. Municipalidad de Guayaquil.

INEC (1996): Cifrando y Descifrando Guayas (Primera Edición) Quito: Instituto Nacional de Estadísticas y Censos INEC.

INEN (2001): Código Ecuatoriano de la Construcción. Quito: INEN.

INEN (1992): DPE INEN 5 Parte 9-1: 1992. (Primera edición) Quito: INEN

Interagua. (2012): Guía de Diseño de Sistemas de Depuración de Aguas Residuales. Guayaquil: Interagua.

Marín Nieto, L. (2011): Mecánica de Suelos. (Sexta edición). Guayaquil: Universidad de Guayaquil.

Núñez Del Arco, E. (2003): Geología del Ecuador. Guayaquil: Escuela Superior Politécnica del Litoral.

Pérez, R. (2002): El Ecuador Profundo (Segunda Edición). Guayaquil: Universidad de Guayaquil.

Pérez, R. (2002): Diccionario Biográfico del Ecuador (Segunda Edición). Guayaquil: Universidad de Guayaquil.

Pérez, R. (1987): Nuestro Guayaquil Antiguo (Tercera Edición). Guayaquil: Universidad de Guayaquil.

PP El Verdadero, (2011): Artículo: Recolección de Desechos en el Guayaquil de Antaño. Edición del 21 de diciembre de 2011.

Ruffilli, A. (2011): Lecciones de Estructuras (Segunda Edición). Guayaquil: Universidad de Guayaquil.

Swyngedouw, Erik; Bovarnick, Andrew (1994): La Crisis de Abastecimiento de Agua en la Ciudad de Guayaquil. (Primera Edición) Guayaquil: Instituto Latinoamericano de Investigaciones Sociales.

Terreros, C. (2011): Tecnología del Hormigón. Guayaquil: Escuela Superior Politécnica del Litoral.

Terreros, C. (2006): Materiales de Construcción. Guayaquil: Escuela Superior Politécnica del Litoral.

Terreros, C. (2011): Investigación de Materiales No Convencionales para la Construcción de Viviendas de Interés Social. Madrid: Universidad Politécnica de Madrid.

Terreros, C. (1988): Los esteros y los problemas de asentamientos en la ciudad de Guayaquil. Guayaquil: Universidad de Guayaquil.

Varela, N. (2013): Estudios para el mejoramiento de los Sistemas de Agua Potable y Alcantarillado Sanitario y Pluvial para la Ciudad de Babahoyo en la Provincia de Los Ríos. Quito: Subsecretaría de Saneamiento Ambiental - MIDUVI. 